Rebecca Dahm

Nathalie Huet

Alexa Craïs

INSPE de Toulouse, Université Toulouse Jean-Jaurès

(France)

\section{Enseigner en contexte de pandémie: quelles différences entre les professeurs du primaire, du secondaire et de l'université?}

\author{
Teaching during the COVID-19 pandemic: what are the differences between
} primary, lower secondary, upper secondary and higher education teachers?

doi: $10.18162 / f p .2021 .743$

\section{ésumé}

Lors de la pandémie liée de COVID-19 du printemps 2020, les enseignants ont été contraints d'assurer la continuité pédagogique en réinventant leur enseignement en dehors de la classe. Par conséquent, le recours aux outils numériques s'est avéré incontournable. Cette recherche évalue, à travers une enquête adressée à 847 enseignants de langue vivante étrangère ou régionale exerçant

dans des établissements français, si le niveau d'enseignement desdits enseignants a un effet sur l'anxiété quilils ressentent, sur leur sentiment

d'efficacité personnelle, ainsi que sur leurs perceptions et usages des outils technologiques en contexte de confinement.

Mots-clés

Sentiment d'efficacité personnelle des enseignants, acceptation des outils technologiques, usages numériques, enseignement des langues vivantes étrangères et régionales, pandémie COVID-19

Abstract

During the Covid 19 pandemic that took place in the spring of 2020, teachers were compelled to ensure pedagogical continuity by reinventing their teaching outside the classroom. As a result, resorting to digital tools became inevitable. This research evaluates, through a survey addressed

to 847 teachers of foreign or regional modern languages working in French schools, whether the level of teaching has an effect on the anxiety these teachers felt, on their sense of self-efficacy, as well as on their perceptions and uses of technological tools in a context of confinement.

\section{Keywords}

Teacher's sense of self-efficacy, technology acceptance, digital usage, teaching of modern foreign and regional languages, COVID-19

\section{Introduction}

Le confinement de ce printemps est tout à fait inédit dans l'histoire de l'humanité et a nécessité une réorganisation de l'enseignement qui s'est effectuée hors de l'institution scolaire. Notre objectif est de comprendre comment les enseignants de langue vivante étrangère ou régionale (LVER) enseignant en contexte scolaire français ont assuré la continuité pédagogique dans le cadre de la crise sanitaire liée à la COVID-19. Or, le métier d'enseignant est très différent selon le niveau scolaire auquel on enseigne. Tout sépare l'école primaire (enfant de 6 à 11 ans) des établissements du secondaire (adolescents âgés de 11 à 17 ans $^{1}$ ) et de l'université : nombre d'élèves, heures d'enseignement, disciplines enseignées, âge des élèves, pratiques d'enseignement, etc. Nous souhaitons donc explorer dans quelle mesure le niveau d'enseignement a une influence sur la mise en place de la continuité pédagogique en période de confinement.

Pour rendre compte de la manière dont les enseignants se sont adaptés, nous avons observé trois grandes variables : l'anxiété, le sentiment d'efficacité personnelle et l'acceptation des outils numériques. En effet, à la suite de la fermeture des établissements scolaires, les enseignants ont été contraints de continuer à exercer leur métier en utilisant des outils numériques. Or, même si depuis plusieurs années, l'usage des outils numériques s'est largement développé, l'acceptation et l'usage de ces nouveaux outils par les enseignants restent problématiques (Berrett, Murphy, \& Sullivan, 2012) et dépendent tant de leur utilité perçue que de leur facilité d'usage.

Davis et ses collègues (1989) définissent l'utilité perçue comme «le degré d'aptitude d'une personne à penser qu'utiliser un système particulier augmentera sa performance au travail». La facilité 
d'utilisation perçue représente «le degré d'aptitude d'une personne à penser qu'utiliser un nouveau système se fera sans efforts ». Comme le souligne Jelassi (2015), il s'agit là du modèle de référence d'acceptation des technologies maintes fois validé empiriquement (Adams et al., 1992). Il a été enrichi à de multiples reprises, des caractéristiques plus affectives (Childers et al., 2001) ou sociales ayant été ajoutées à celui-ci (Venkatesh et Davis, 2000). Or, c'est le caractère social du modèle qui nous intéresse particulièrement dans le contexte de mise en place de la continuité pédagogique. Selon le modèle de Vankatesh et Bala (2008), l'utilité perçue et la perception de facilité d'usage permettent de prédire la manière dont l'enseignant va s'emparer des outils numériques.

On sait également que le sentiment d'efficacité personnelle dans l'usage des nouveaux outils affecte indirectement leur utilisation (Venkatesh et Bala, 2008). Ce concept développé par Bandura (1977) renvoie à l'évaluation subjective de l'individu sur ses propres capacités à accomplir une tâche donnée et détermine les performances par ses effets tant sur la quantité d'effort que sur la qualité de l'effort (Bandura, 1986). Galand et Vanlede (2004), qui ont recensé un grand nombre de travaux, soulignent l'existence d'une relation entre sentiment d'efficacité personnelle et performance ou persévérance.

Pour Tschannen-Moran, Hoy, et Hoy (1998), le SEP est spécifique à l'activité ou au domaine dans lequel il est perçu. Plusieurs études ont été menées pour déterminer le SEP des enseignants, qu'ils soient enseignants généralistes novices (Tschannen-Moran et Woolfolk Hoy (2007)) ou experts (ibid. ; Duffy et al. (2012)) ou spécialistes (Olsommer et Voymae-egger (2015). D'autres travaux (e.g. Gaudreau, Royer, Beaumont \& Frenette, 2012; Gibson \& Dembo, 1984) montrent aussi que les enseignants présentant un SEP élevé modifient et adaptent leur pédagogie, quelles que soient les difficultés des élèves ou la complexité de la situation, créant ainsi des conditions favorables à l'apprentissage, contrairement à ceux ayant un SEP faible. Le SEP des enseignants a donc un impact non seulement sur l'usage des nouveaux outils numériques, mais aussi sur l'apprentissage de l'élève.

Explorer le concept de sentiment de compétence des enseignants en matière d'intégration des outils numériques est également intéressant. En effet, il s'inscrit aussi dans l'approche socio-cognitive (Rey, 2013) et permet de traduire la perception des individus à accomplir certaines tâches (Pintrich et Schunk, 2002). Tout comme le SEP, il peut influencer la volonté de s'engager dans une tâche et l'attrait de la tâche (Seegers et Boekaerts, 1993).

Dans ce cadre, l'objet de la présente recherche est d'évaluer si l'anxiété, le SEP et les perceptions des outils numériques ainsi que leurs usages varient selon le niveau d'enseignement, lors de la mise en place de la continuité pédagogique en contexte de confinement.

\section{Matériel et méthode}

L'enquête a été menée lors de la période de confinement qui a eu lieu en France du 16 mars au 11 mai 2020 en raison de la pandémie mondiale de COVID-19. Le lien de l'enquête, disponible du 23 avril au 10 mai 2020, a été transféré par le biais de la liste des enseignants de langue appartenant à la base de données de la direction des services de l'éducation nationale de Toulouse (appelé Rectorat) ainsi que par la messagerie de différentes sociétés savantes; le lien a également été publié sur le site de l'association des professeurs de langue vivante (APLV) et sur le site du réseau des établissements universitaires de formations des enseignants, les Instituts Nationaux Supérieurs du Professorat et de l'Éducation (INSPE).

2 - Formation et profession $28(4$ hors-série), 2020 
Le questionnaire comprenant 38 questions et 98 items nécessitait environ vingt minutes pour être rempli. Sur les 1361 répondants ayant consulté le questionnaire, 748 ont complété l'ensemble des données nous permettant de constituer l'échantillon. Les coefficients alpha de Cronbach des items intra-variable (et dimension de variable) étant supérieurs à 0,80, la cohérence interne est satisfaisante.

\section{Participants et caractéristiques des niveaux d'enseignement}

Les données utilisées pour cette recherche ont été récoltées auprès d'enseignants de langue exerçant dans des établissements français de métropole $(\mathrm{n}=707)$, d'outremer $(\mathrm{n}=36)$ ou de l'étranger $(\mathrm{n}=5)$. La population à l'étude $(\mathrm{n}=748)$ est composée d'enseignants qui enseignent en cycle primaire $(\mathrm{n}=29)$ et ont des élèves âgés de 3 à 11 ans en moyenne, d'enseignants qui enseignent en cycle secondaire au collège $(\mathrm{n}=305)$, à des élèves âgés de 12 à 15 ans en moyenne, d'enseignants qui enseignent en cycle secondaire, au lycée en France ( $n=317)$, qu'il soit général, technologique ou professionnel et qui enseignent à des élèves âgés de 16 à 18 ans en moyenne ainsi que d'enseignants qui enseignent à l'université $(\mathrm{n}=97)$ à des étudiants âgés de 18 ans et plus.

\section{Tableau 1}

Composition de l'échantillon (niveau d'enseignement, genre et langue enseignée)

\begin{tabular}{|c|c|c|c|c|c|c|}
\hline & Niveau & primaire & collège & lycée & université & TOTAL \\
\hline & $\mathrm{n}=$ & 29 & 304 & 317 & 97 & 748 \\
\hline \multirow{3}{*}{ Genre } & Homme & 1 & 33 & 28 & 16 & 78 \\
\hline & femme & 28 & 272 & 287 & 81 & 668 \\
\hline & Non renseigné & & & 2 & & 2 \\
\hline \multirow{8}{*}{$\begin{array}{l}\text { Langue(s) } \\
\text { enseignées }\end{array}$} & allemand & 1 & 31 & 27 & 5 & 64 \\
\hline & anglais & 24 & 218 & 203 & 78 & 523 \\
\hline & chinois & & 1 & 2 & & 3 \\
\hline & espagnol & & 33 & 57 & 10 & 100 \\
\hline & italien & & 15 & 27 & 3 & 45 \\
\hline & langue régionale & 4 & 7 & 1 & & 12 \\
\hline & FLE/FLS & & & & 1 & 1 \\
\hline & $\begin{array}{l}\text { Deux langues ou } \\
\text { plus }\end{array}$ & 4 & 4 & 4 & 3 & 15 \\
\hline
\end{tabular}

Sur l'ensemble de la population, $70 \%$ enseignent l'anglais ( $\mathrm{n}=523), 13 \%$ l'espagnol $(\mathrm{n}=100), 9 \%$ l'allemand $(n=64), 6 \%$ l'italien $(n=45), 1,2 \%$ la langue régionale $(n=12), 0,4 \%$ le chinois $(n=3)$ et $0,13 \%$ le français langue étrangère/langue seconde $(n=3)$. Néanmoins, dans le cadre du présent article, nous choisissons de ne pas effectuer de distinction entre les langues étudiées et d'envisager la population comme une population homogène d'enseignants de langue, partageant des préoccupations communes. 
$89,3 \%$ des répondants sont des enseignantes $(n=668)$, alors que leurs collègues masculins $(n=78)$ ne constituent que 10,4\% de notre échantillon. Deux participants n’ont pas donné d'indication genrée.

Niveau d'enseignement et anxiété des enseignants

Cinq questions permettent de connaître l'état d'anxiété générale et sanitaire (anxiété liée aux bouleversements des conditions de vie, à la santé personnelle ou celle des proches) ou professionnelle : maintien de la continuité pédagogique, relations avec les étudiants/élèves et les parents, relations avec la hiérarchie (personnel de direction, inspection) ainsi que l'anxiété ressentie par rapport à la reprise des cours le 11 mai en conformité avec le respect des conditions de sécurité sanitaire. Elles sont évaluées par le degré d'accord de l'enseignant avec chaque affirmation sur une échelle de (1) très serein à (7) très anxieux.

\section{Niveau d'enseignement et sentiment d'efficacité personnelle relatif à la continuité pédagogique}

Le SEP des enseignants relatif à la mise en place de la continuité pédagogique est évalué à travers 12 items issus de la version française (De Stercke et al., 2014) du questionnaire de Tschannen-Moran et Woolfolk-Hoy (2001), le Teachers' Sense of Efficacy Scale (TSES), bénéficiant d'une validité éprouvée dans des contextes culturels divers (Klassen et al., 2009). De plus, comme le soulignent de nombreux auteurs (e.g., Valls et Bonvin, 2015), le choix de ce questionnaire est d'autant plus pertinent qu'il refléterait le mieux la définition de Bandura (1997) du SEP. Enfin, c'est une des échelles les plus utilisées dans les recherches actuelles évaluant le SEP des enseignants.

Nous avons réutilisé le TSES en l'adaptant au contexte du confinement par l'ajout de termes tels que «à distance». Ce questionnaire reflète trois dimensions distinctes des pratiques d'enseignement à distance : les stratégies d'enseignement (4 items), la gestion de classe (4 items) et l'engagement des élèves (4 items). Nous avons également ajouté neuf items permettant de mesurer le sentiment de compétence numérique perçu dans le cadre de l'enseignement de la LVER à distance (capacité à travailler les neuf compétences suivantes : compréhension de l'écrit, compréhension de l'oral, expression écrite en continu, expression écrite en interaction, expression orale en continu, expression orale en interaction, compétence phonologique, grammaticale, lexicale). Pour l'ensemble des items, le participant devait se positionner sur une échelle allant de Absolument pas capable (0) à Absolument capable (9), selon le principe établi par Tschannen-Moran (2001).

\section{Niveau d'enseignement et utilisation des outils technologiques pour assurer la continuité pédagogique}

L'acceptation des outils numériques pour assurer la continuité pédagogique est évaluée en prenant en compte à la fois l'utilité perçue et la facilité d'utilisation. Les questions prennent appui sur les items utilisés par Davis (1989) et précisent qu'ils portent sur l'enseignement en période de confinement.

Pour la perception de facilité d'utilisation des outils numériques pendant le confinement (3 items), l'enseignant est amenéà indiquer son degré d'accord relatifà la facilité d'usage des outils numériques dans le cadre de la mise en œuvre de son enseignement (clarté, intuitivité, faible effort mental, construction d'enseignement interactif) sur une échelle de (1) pas du tout d'accord à (7) tout à fait d'accord.

4 - Formation et profession 28(4 hors-série), 2020 
L'utilité perçue des outils numériques est évaluée par 16 items. Pour chaque item, l'enseignant doit indiquer son degré d'accord (même échelle que précédemment) relatif à l'utilité des outils numériques, que ce soit pour travailler les compétences avec les élèves ou pour sa pratique professionnelle (préparation des cours, collaboration avec les collègues, relation avec les élèves).

L'usage des outils numériques (10 items) est également évalué pendant le confinement. Les enseignants sont invités à cocher les outils numériques qu'ils utilisent pour le maintien de la continuité pédagogique (Classe virtuelle (ou tout type de visioconférence), tchats (SMS, WhatsApp, etc.), forums, mails, téléphone, manuel numérique, l'espace numérique de travail de l'établissement, supports préexistants sur des sites extérieurs (Eduscol, Learning Apps, Youtube, etc), supports créés, déposés ou recueillis sur ses "pages» perso (padlets, pearltrees, Learning Apps, chaîne perso YouTube, etc.) pour travailler les compétences suivantes : compréhension de l'écrit, expression écrite en continu, expression écrite en interaction, expression orale en continu, expression orale en interaction, prononciation, compétence grammaticale, compétence lexicale. Dans la présente étude, nous avons relevé le nombre de fois où chaque outil est utilisé (étendue des réponses allant de 0 (jamais utilisé) à 8 (utilisé pour travailler l'ensemble des compétences)).

\section{Présentation des résultats}

À l'aide de SPSS (version 21.0), nous avons d'abord examiné les moyennes, les écarts types et la distribution des fréquences de chacun des items. Une analyse de la variance unidirectionnelle one-way ANOVA et post hoc de Tukey a été menée pour chaque ensemble d'items (anxiété, SEP, perceptions et outils) de manière à établir une relation entre le niveau d'enseignement et les variables indiquées.

\section{Niveau d'enseignement et anxiété des enseignants}

Nous avons pris en compte les réponses aux cinq questions permettant de connaître l'anxiété générale et sanitaire et l'anxiété professionnelle, ce qui nous permet de déterminer l'anxiété ressentie lors de la mise en place de la continuité pédagogique. L'échelle de réponse s'inscrit entre les bornes 1 et 7, ce qui nous permet d'obtenir les moyennes suivantes :

\section{Tableau 2}

Moyennes, écarts types et analyses unidirectionnelles de la variance de l'anxiété en contexte de confinement en fonction du niveau d'enseignement

\begin{tabular}{|c|c|c|c|c|c|c|c|c|c|c|}
\hline \multirow[t]{2}{*}{ Mesure } & \multicolumn{2}{|c|}{ Primaire } & \multicolumn{2}{|c|}{ Collège } & \multicolumn{2}{|c|}{ Lycée } & \multicolumn{2}{|c|}{ Université } & \multirow[t]{2}{*}{$F(3,744)$} & \multirow[t]{2}{*}{ n2 } \\
\hline & M & SD & M & SD & M & SD & M & SD & & \\
\hline $\begin{array}{l}\text { Anxiété générale } \\
\text { et sanitaire }\end{array}$ & 4,45 & 1,64 & 4,31 & 1,64 & 4,49 & 1,51 & 3,95 & 1,49 & $3,06^{*}$ & 7,53 \\
\hline $\begin{array}{l}\text { Anxiété } \\
\text { professionnelle }\end{array}$ & 4,48 & 1,36 & 4,61 & 1,36 & 4,79 & 1,32 & 4,16 & 1,50 & $5,08^{* *}$ & 1,85 \\
\hline $\begin{array}{l}\text { Anxiété continuité } \\
\text { pédagogique }\end{array}$ & 4,47 & 1,25 & 4,50 & 1,30 & 4,68 & 1,24 & 4,08 & 1,36 & $5,12^{* *}$ & 8,37 \\
\hline \multicolumn{11}{|c|}{ * . La différence moyenne est significative au niveau 0.05 . } \\
\hline ** La différence mo & est si & tive at & au 0.0 & & & & & & & \\
\hline
\end{tabular}


Afin de comparer les moyennes entre les différents groupes, nous avons effectué une analyse de la variance (ANOVA) à un facteur. Celle-ci montre un effet significatif du niveau d'enseignement sur l'anxiété ressentie lors de la mise en ouvre de la continuité pédagogique $(\mathrm{F}(3,744)=5,12 ; \mathrm{p}=0,002)$. L'effet est également significatif pour les deux dimensions qui la composent : l'anxiété générale et sanitaire $(\mathrm{F}(3,744)=3,06 ; \mathrm{p}=0,028)$ ainsi que l'anxiété professionnelle $(\mathrm{F}(3,744)=5,08 ; \mathrm{p}=0,002)$.

L'analyse post hoc avec le test de Tukey fait apparaître une différence significative entre les groupes d'enseignants dans les classes du secondaire (collège) et ceux du supérieur ( $\mathrm{HSD}=0,417 ; \mathrm{p}=0,035$ ) ainsi qu'entre les groupes d'enseignants du secondaire (lycée) et ceux du supérieur (HSD = 0,596; $p=$ 001), lorsqu'on examine l'anxiété ressentie lors de la mise en œuvre de la continuité pédagogique.

Lors de l'analyse des deux dimensions qui la composent, on observe des relations similaires pour l'anxiété professionnelle : le post-hoc fait apparaître un HSD $=0,450$ avec $p=0,032$ pour les enseignants du secondaire en collège et du supérieur ainsi qu'un HSD $=0,628$ avec $p=0,001$ pour les enseignants du secondaire en lycée et les enseignants du supérieur. Cependant, la différence n'est significative qu'entre les enseignants du lycée et ceux du supérieur $(\mathrm{HSD}=0,4541 ; \mathrm{p}=0,016)$ pour l'anxiété générale et sanitaire.

Autrement dit, les enseignants du supérieur ont ressenti significativement moins d'anxiété professionnelle que les deux groupes d'enseignants du secondaire. Les enseignants de lycée ont ressenti significativement plus d'anxiété générale et sanitaire que les enseignants du supérieur. On ne peut cependant pas relever de différence significative entre les professeurs du cycle primaire et les trois autres groupes relative à l'anxiété, qu'elle soit générale et sanitaire, professionnelle ou liée de manière globale au contexte de mise en œuvre de la continuité pédagogique.

\section{Niveau d'enseignement et sentiment d'efficacité personnelle}

Douze questions ont été posées dans le but de connaître le sentiment d'efficacité personnelle des enseignants (Tschannen-Moran, 2001). Elles peuvent être regroupées selon trois dimensions, à savoir les stratégies d'enseignement, la gestion de la classe et l'engagement des élèves/étudiants. Nous avons choisi d'y ajouter également le sentiment de compétence qui permet d'informer le SEP lié à la mise en œuvre de la continuité pédagogique. 


\section{Tableau 3}

Moyennes, écarts types et analyses unidirectionnelles de la variance du SEP et de ses dimensions en contexte de confinement selon le niveau d'enseignement

\begin{tabular}{lcccccccccc}
\hline Mesure & \multicolumn{2}{c}{ Primaire } & \multicolumn{2}{c}{ Collège } & \multicolumn{2}{c}{ Lycée } & \multicolumn{2}{c}{ Université } & F(3,744) & n2 \\
\hline & $M$ & SD & $M$ & SD & $M$ & SD & $M$ & SD & & \\
\hline SEP-Stratégies & 5,34 & 2,02 & 5,84 & 1,89 & 5,92 & 1,89 & 5,78 & 1,98 & 0,90 & 3,30 \\
\hline SEP-Gestion & 4,35 & 2,50 & 4,02 & 2,14 & 4,26 & 2,16 & 4,14 & 2,15 & 0,73 & 3,42 \\
\hline SEP-Engagement & 5,83 & 1,86 & 5,59 & 1,73 & 5,22 & 1,78 & 4,28 & 1,52 & $15,17^{* *}$ & 45,63 \\
\hline $\begin{array}{l}\text { SEP } \\
\text { Sentiment de } \\
\text { Compétence }\end{array}$ & 3,61 & 1,97 & 5,75 & 1,34 & 5,94 & 1,46 & 5,28 & 2,10 & $23,27^{*}$ & 54,58 \\
\hline
\end{tabular}

** La différence moyenne est significative au niveau 0.005 .

L'analyse de la variance (ANOVA) à un facteur ne montre pas d'effet significatif du niveau d'enseignement sur le SEP lié à la continuité pédagogique (moyenne de l'ensemble des réponses); néanmoins, un effet significatif apparaît lorsqu'on examine la dimension engagement $(F(3,744)=15,17 ; p=0,000)$. L'analyse post hoc menée avec le test de Tukey montre une différence significative entre les groupes enseignants du supérieur et les enseignants de primaire (HSD $=-1,549 ; \mathrm{p}=0,000)$ ainsi qu'entre les enseignants du secondaire en collège ( $\mathrm{HSD}=-1,313 ; \mathrm{p}=0,000)$ et les enseignants du secondaire en lycée (HSD = -0,938; $\mathrm{p}=0,000)$. Les enseignants du secondaire en collège ont l'impression de davantage parvenir à engager leurs élèves que les enseignants du lycée, et ce, de manière significative. En revanche, les enseignants du supérieur parviennent significativement moins à engager leurs élèves/ étudiants que les trois groupes d'enseignants, qu'ils soient du secondaire ou du primaire.

De la même façon, un effet significatif du niveau d'enseignement sur le sentiment de compétence apparaît $(F(3,744)=23,268 ; p=0,000)$. L'analyse post hoc avec le test de Tukey montre une différence significative entre le groupe professeurs du primaire et l'ensemble des trois groupes : enseignants de collège ( $\mathrm{HSD}=-2,145 ; \mathrm{p}=0,000)$, enseignants de lycée $(\mathrm{HSD}=-2,336 ; \mathrm{p}=0,000)$ et enseignants du supérieur $(\mathrm{HSD}=-1,665 ; \mathrm{p}=0,000)$. Les professeurs du primaire se sentent significativement moins compétents que les autres enseignants pour utiliser les outils numériques lors de l'enseignement de la langue vivante. A contrario, les enseignants de collège et de lycée se sentent plus compétents que les enseignants du supérieur (respectivement $\mathrm{HSD}=0,473 ; p=0,041$ et $\mathrm{HSD}=0,665 ; \mathrm{p}=0,001$ ), même si ces derniers se sentent tout de même plus compétents que les professeurs des écoles. 


\section{Niveau d'enseignement et acceptation des technologies}

L'analyse des réponses obtenues aux seize items permettant d'informer la perception d'utilité de l'outil numérique par l'ensemble des enseignants (échelle de Likert allant de 1 à 7 ) fait apparaître les données suivantes :

\section{Tableau 4}

Moyennes, écarts types et analyses unidirectionnelles de la variance de l'acceptation des outils numériques et de ses dimensions en contexte de confinement selon le niveau d'enseignement

\begin{tabular}{|c|c|c|c|c|c|c|c|c|c|c|}
\hline \multirow[t]{2}{*}{ Mesure } & \multicolumn{2}{|c|}{ Primaire } & \multicolumn{2}{|c|}{ Collège } & \multicolumn{2}{|c|}{ Lycée } & \multicolumn{2}{|c|}{ Université } & \multirow[t]{2}{*}{$F(3,744)$} & \multirow[t]{2}{*}{$\mathrm{n} 2$} \\
\hline & M & SD & M & SD & M & SD & $M$ & SD & & \\
\hline Utilité perçue & 4,85 & 1,23 & 4,71 & 1,02 & 4,53 & 1,06 & 4,35 & 1,04 & $4,10^{* *}$ & 4,51 \\
\hline $\begin{array}{l}\text { Facilité d'utilisation } \\
\text { perçue }\end{array}$ & 4,23 & 1,46 & 3,95 & 1,48 & 3,69 & 1,63 & 3,53 & 1,50 & $3,18^{* *}$ & 7,59 \\
\hline $\begin{array}{l}\text { Perception outils } \\
\text { numériques }\end{array}$ & 4,45 & 1,38 & 4,51 & 1,03 & 4,32 & 1,12 & 4,13 & 1,11 & $3,59^{*}$ & 4,28 \\
\hline \multicolumn{11}{|c|}{ *. La différence moyenne est significative au niveau 0.05.} \\
\hline ** La différence moy & nne est & ificativ & niveau & & & & & & & \\
\hline
\end{tabular}

L'analyse de la variance (ANOVA) à un facteur montre un effet significatif du niveau d'enseignement sur l'utilité perçue $(\mathrm{F}(3,744)=4,10 ; \mathrm{p}=0,007)$, sur la facilité d'utilisation perçue $((\mathrm{F}(3,744)=3,18 ; \mathrm{p}$ $=0,002)$ ainsi que sur l'acceptation des outils numériques $(F(3,744)=3,59 ; \mathrm{p}=0,013)$.

L'analyse post hoc avec le test de Tukey souligne une différence significative entre les groupes d'enseignants de collège et ceux du supérieur ( $\mathrm{HSD}=0,367 ; \mathrm{p}=0,015)$, en ce qui concerne l'utilité perçue. Les enseignants de collège ont donc une perception de l'utilité d'utilisation significativement supérieure à celle des enseignants du supérieur. On retrouve également une différence significative entre enseignants du secondaire en collège et du supérieur, en ce qui concerne l'acceptation des outils numériques (HSD = 0,386; $\mathrm{p}=0,013)$. Selon Venkatesh et Bala (2008), ces éléments nous amènent à prédire que les enseignants du secondaire en collège utiliseront plus aisément un grand nombre d'outils pour enseigner les différentes compétences que les enseignants du supérieur.

L'analyse des outils numériques utilisés pour travailler les compétences régulièrement utilisées en classe de langue en contexte de confinement selon le niveau d'enseignement fait apparaître les données suivantes ( $\max =8$ soit le nombre de fois où l'outil est choisi pour travailler une compétence). 


\section{Tableau 5}

Moyennes, écarts types et analyses unidirectionnelles de la variance d'usage des outils numériques en contexte de confinement selon le niveau d'enseignement

\begin{tabular}{|c|c|c|c|c|c|c|c|c|c|c|}
\hline \multirow[t]{2}{*}{ Mesure } & \multicolumn{2}{|c|}{ Primaire } & \multicolumn{2}{|c|}{ Collège } & \multicolumn{2}{|c|}{ Lycée } & \multicolumn{2}{|c|}{ Supérieur } & \multirow[t]{2}{*}{$F(3,744)$} & \multirow[t]{2}{*}{$\mathrm{n} 2$} \\
\hline & M & SD & $M$ & SD & M & SD & $M$ & SD & & \\
\hline Classe virtuelle & 1,03 & 1,64 & 1,34 & 2,07 & 2,23 & 2,43 & 2,26 & 2,43 & $10,30^{* *}$ & 52,68 \\
\hline Tchats, SMS,... & 0,24 & 0,95 & 0,36 & 1,12 & 0,75 & 1,53 & 0,47 & 1,24 & $5,31^{* *}$ & 9,23 \\
\hline Forums & 0,00 & 0,00 & 0,17 & 0,76 & 0,19 & 0,72 & 0,51 & 1,37 & $4,94^{* *}$ & 3,44 \\
\hline Mails & 1,90 & 1,93 & 1,84 & 2,07 & 2,84 & 2,18 & 1,94 & 1,88 & $13,30^{* *}$ & 57,89 \\
\hline Téléphone & 0,07 & 0,37 & 0,21 & 0,85 & 0,37 & 0,95 & 0,23 & 0,65 & 2,36 & 1,75 \\
\hline Manuel num & 0,14 & 0,44 & 1,23 & 1,89 & 1,16 & 1,76 & 0,11 & 0,68 & $14,38^{* *}$ & 40,74 \\
\hline $\begin{array}{l}\text { Espace numérique } \\
\text { de travail }\end{array}$ & 0,90 & 1,65 & 3,61 & 2,38 & 3,15 & 2,29 & 1,93 & 2,14 & $22,47^{* *}$ & 117,63 \\
\hline Supports existants & 1,14 & 1,83 & 1,58 & 2,03 & 1,26 & 1,85 & 0,55 & 1,32 & $7,70^{* *}$ & 26,77 \\
\hline Supports perso & 1,45 & 2,15 & 2,25 & 2,37 & 1,67 & 2,24 & 0,78 & 1,72 & $11,38^{* *}$ & 56,62 \\
\hline autre & 0,17 & 0,54 & 0,47 & 1,21 & 0,41 & 1,09 & 0,30 & 0,99 & 1,05 & 1,30 \\
\hline Moy. des outils & 0,70 & 0,50 & 1,31 & 0,70 & 1,40 & 0,69 & 0,91 & 0,68 & $19,72^{* *}$ & 9,31 \\
\hline
\end{tabular}

** La différence moyenne est significative au niveau 0.005 .

Les outils les plus utilisés par l'ensemble des enseignants sont les espaces numériques de travail institutionnalisés (moy $=3,088)$, les mails $(\operatorname{moy}=2,275)$, la classe virtuelle $(\mathrm{moy}=1,825)$ ainsi que les supports ou pages personnelles (moy=1,780).

L'analyse de la variance (ANOVA) à un facteur montre un effet significatif $(p=0,000)$ du niveau d'enseignement sur l'usage des outils, et ce, quel que soit l'outil utilisé (moyenne des outils : $(\mathrm{F}(3,744)$ $=9,31 ; p=0,000)$. Même si le nombre moyen d'utilisations reste faible et que l'écart-type est élevé, on observe que ce sont les enseignants du secondaire en lycée qui ont le plus fréquemment recours aux outils numériques pour le maintien de la continuité pédagogique, peu importe l'outil utilisé (Moyenne des outils $=1,40)$.

L'analyse post hoc avec le test de Tukey fait apparaître un certain nombre de différences significatives entre les quatre groupes d'enseignants. On observe que les collègues du secondaire (collège et lycée) utilisent significativement plus d'outils numériques que les collègues du primaire ou du supérieur. La distinction se remarque surtout sur le plan de l'utilisation de l'espace numérique de travail et du manuel numérique, deux outils essentiellement utilisés dans le secondaire et plus particulièrement au collège.

Même si les mails sont utilisés par tous pour mettre en place la continuité pédagogique, une différence significative apparaît pour la communication avec les élèves les plus grands : en effet, les enseignants du supérieur l'utilisent significativement plus que les enseignants du secondaire en lycée (HSD=0,102 ; $\mathrm{p}=0,000$ ), même si ces derniers l'utilisent significativement plus que les enseignants du secondaire en collège $(\mathrm{HSD}=1 ; \mathrm{p}=0,000)$. Il en va de même pour la classe virtuelle (ou tout autre type de support de visioconférence) : plus on avance en âge, plus la différence devient significative. 


\section{Discussion et conclusion}

Les analyses menées ci-dessus nous permettent de mieux circonscrire le SEP relatif à la mise en place de la continuité pédagogique. On remarque que pour l'ensemble de l'échantillon, les résultats indiquent une moyenne de 5,1 sur 9 au score global du SEP, correspondant à la position médiane «ni capable, ni pas capable» sur l'échelle de mesure du SEP. Or, Tschannen-Moran et Woolfolk Hoy (2007) ayant mené une étude en contexte américain obtiennent un score global de 6,87 pour les enseignants débutants et de 7,29 pour les enseignants expérimentés. Duffy et al. (2012) rapportent un score global de 6,69 pour des enseignants débutants. Dans l'étude menée par Olsommer et Voyame-Egger (2015) auprès d'enseignants spécialisés en formation, le score obtenu est de 6,2. Par conséquent, qu'il s'agisse d'enseignants spécialisés débutants ou de généralistes expérimentés, les scores obtenus sont largement supérieurs à ceux de notre population d'enseignants de langue, mesurés en contexte de confinement.

Pourtant, pour Viel-Ruma et al. (2010) ainsi que pour Gaudreau et al. (2012), la satisfaction du travail des enseignants généralistes est supérieure à ceux travaillant avec des élèves présentant des troubles émotionnels et du comportement. On pouvait ainsi s'attendre à obtenir, pour notre population d'enseignants généralistes, un SEP supérieur à celui des enseignants spécialisés. Néanmoins, Caprara et al. (2003) soulignent à quel point le SEP des enseignants est directement lié à leur satisfaction au travail, si bien qu'il devient un élément décisif influençant les attitudes et les performances des enseignants. Or, comment ressentir de la satisfaction au travail dans le contexte inédit de confinement, devant l'aspect soudain des mesures prises et à l'absence d'expérience antérieure? Il va sans dire que ces éléments ont eu un effet sur l'état émotionnel de l'enseignant, l'une des sources du SEP (Bandura, 1986).

L'analyse menée a permis d'observer que ce sont avant tout les enseignants du secondaire et plus particulièrement du lycée qui ont ressenti l'anxiété la plus forte, qu'elle soit professionnelle ou générale. A contrario, les enseignants du supérieur ont moins été soumis à l'anxiété professionnelle, et ce, de manière significative. Il est fort probable que le stress ressenti par les enseignants de lycée en amont du confinement était déjà plus élevé, puisqu'une réforme majeure de l'enseignement secondaire était en cours de mise en œuvre. En outre, pour Dejan et Charlier (2011), le manque de reconnaissance dont souffrent les enseignants débutants durant leurs premières années sur le terrain affecte négativement le SEP; même si la population de notre étude est composée d'enseignants à profils diversifiés, on peut se demander si le côté inédit du confinement ne les place pas tous dans la situation de l'enseignant débutant qui tâtonne et doit découvrir par lui-même de nouvelles manières de procéder. Plusieurs études soulignent la corrélation positive qui existe entre le SEP et l'expérience professionnelle (Benoît, 2013; Klassen et Chiu 2010; Wolters \& Daugherty, 2007). On peut ainsi penser que le faible SEP obtenu dans notre étude reflète la manière dont le confinement a affecté la confiance des enseignants dans leur efficacité à mettre en œuvre la continuité pédagogique.

Lorsqu'on affine l'analyse en prenant appui sur les études de Tschannen-Moran (2007; n= 181), Duffy $(2012 ; n=272)$ et Olsommer et Voyame-Egger (2015; n=30), on observe qu'habituellement, la gestion de classe est la dimension avec le score le plus élevé. Or, dans notre étude, il s'agit du score le plus faible, bien en deçà du score moyen obtenu au SEP. Ce résultat semble cohérent en contexte de confinement puisque les nouvelles modalités de mise en ouvre (classe virtuelle, par exemple) rendent 
difficile l'interaction directe permettant de gérer la classe et de maintenir la discipline. De plus, les scores obtenus ne sont pas significativement différents entre les groupes d'enseignants; on peut dès lors penser que la gestion de classe a été vécue de la même façon par l'ensemble des enseignants, en contexte de confinement.

Dans le cadre de notre étude, c'est la capacité à engager les élèves qui présente le plus de différence significative entre les niveaux d'enseignement: le score baisse, au fur et à mesure que l'on avance en âge, et la différence demeure significative entre les quatre groupes, ce qui laisse à penser que les enseignants ont plus de facilité à maintenir l'engagement des jeunes élèves lors du maintien de la continuité pédagogique.

Finalement, le score obtenu pour les stratégies d'enseignement est le plus élevé même s'il n'est pas significativement différent entre les groupes. On peut dès lors penser que les enseignants ont réussi à adapter les stratégies d'enseignement au contexte nouveau, quel que soit le niveau auquel ils enseignent, et ce, malgré la contrainte imposée par les outils numériques.

Tricot et al. (2003) mettent en avant le fait que les outils numériques ne seront utilisés que s'ils sont acceptés, utilisables et utiles. On peut toutefois penser que la situation de confinement a modifié les représentations personnelles des enseignants de ce qu'ils considèrent comme «une technologie adaptée à leur activité, une pédagogie efficace, des contenus didactiques efficients, des stratégies cognitives performantes» (Trestini, 2012). Les résultats obtenus dans le cadre de l'étude de l'utilité perçue et de la facilité d'utilisation perçue des outils numériques, inscrite dans le modèle de Venkatesh et Bala (2008), ont permis de montrer que l'ensemble des enseignants considèrent les outils comme utiles pour enseigner en contexte de confinement; néanmoins, tant l'utilité perçue que la facilité d'usage décroissent au fur et à mesure que l'on avance dans l'enseignement. On s'attendrait donc à ce que ce soient les enseignants du primaire qui aient le plus recours aux outils numériques. Or, ils utilisent significativement moins d'outils pour l'enseignement de la langue en contexte de confinement que leurs collègues du secondaire. Cette étude met à nouveau en exergue le cas particulier des enseignants du supérieur qui considèrent significativement les outils numériques comme moins utiles que les enseignants du secondaire en collège. De la même manière, ils ont moins eu recours aux outils numériques pour maintenir la continuité pédagogique que leurs collègues du secondaire. Il est fort probable que l'utilisation de l'espace numérique de travail au quotidien voire des manuels numériques ainsi que l'offre d'outils institutionnels aient permis à ces derniers de rapidement s'adapter au nouveau contexte, de manière à continuer à travailler les compétences de communication et linguistiques propres à l'enseignement/apprentissage des LVER.

En conclusion, il nous semble possible d'affirmer que le niveau d'enseignement a bien eu un effet de manière différente les quatre groupes d'enseignants, que ce soit sur le plan de l'anxiété, du SEP ou de l'acceptation des technologies. Nous avons également pu valider le modèle de Venkatesh et Bala (2008) qui nous a permis de prédire les observations relatives aux usages du numérique en contexte de confinement et de vérifier que ces derniers varient en fonction du niveau auquel s'adresse l'enseignant. Nous avons ainsi pu observer à quel point le SEP des enseignants issus du premier degré, du secondaire ou du supérieur était faible lors de la mise en place soudaine de la continuité pédagogique en France, au printemps 2020, à la suite de la pandémie liée à la COVID-19. Par conséquent, si on souhaite mieux les préparer à de nouveaux bouleversements éventuels, il nous semble essentiel de renforcer leur SEP 
par des retours positifs de la hiérarchie, par la mise en place de réseaux facilitant le partage d'expérience et par des formations adaptées à l'enseignement à distance.

\section{Note}

1 Le cycle secondaire en France se divise en deux cycles : le collège unique qui accueille tous les élèves âgés entre 11 et 15 ans et le lycée plus sélectif pour les enfants de 15 à 17 ans.

\section{Références}

Adams D., Nelson R.-R. Et Todd P. (1992), Perceived usefulness, ease of use and usage of information technology: a replication, MIS Quaterly, 16(2), 227-248.

Bandura, A. (1977). Self-efficacy : toward a unifying theory of behavioral change. Psychological review, 84(2), 191.

Bandura, A. (1986). The explanatory and predictive scope of self-efficacy theory. Journal of social and clinical psychology, 4(3), 359-373.

Bandura, A. (1988). Self-efficacy conception of anxiety. Anxiety research, 1(2), 77-98.

Bandura, a. (1997). Self-efficacy : The exercise of control. New York, New York : Freeman.

Berrett, B., Murphy, J., \& Sullivan, J. (2012). Administrator insights and reflections: Technology integration in schools. Qualitative Report, 17(1), 200-221.

Caprara, G. V., Barbaranelli, C., Borgogni, L., Steca, P. (2003). Efficacy beliefs as determinants of teachers' job satisfaction. Journal of educational psychology, 95(4), 821-832.

Clément, L., Fernet, C., Morin, A. J. S., \& Jeanrie, C. (2018). Validation canadienne-française de l'Échelle des déterminants du sentiment d'efficacité personnelle des enseignants (EDSEPE). Psychologie du Travail et des Organisations, 24(2), 126-143.

Compeau, D. R., \& Higgins, C. A. (1995). Computer self-efficacy : Development of a measure and initial test. MIS quarterly, 19(2), 189-211.

Davis, F. D. (1989). Perceived usefulness, perceived ease of use, and user acceptance

of information technology. MIS Quarterly, 13(3), 319-340.

Davis F.-D., Bagozzi R. et Warshaw P.-R. (1989), User acceptance of computer technology : a comparison of two theoretical models, Management Science, 35(8), 982-1003.

De Stercke, J., Temperman, G., De Lièvre, B., \& Lacocque, J. (2014). Echelle de sentiment

d'efficacité personnelle des enseignants : traduction francophone de la Teachers' Sense of

Efficacy Scale. Service de Pédagogie Générale et des Médias Educatifs, Université de Mons.

Duffin, L. C., French, B. F., \& Patrick, H. (2012). The Teachers' Sense of Efficacy Scale: Confirming the factor structure with beginning pre-service teachers. Teaching and teacher Education, 28(6), 827-834.

Gaudreau, N., Royer, É., Beaumont, C., \& Frenette, É. (2012). Le sentiment d'efficacité personnelle des enseignants et leurs pratiques de gestion de la classe et des comportements difficiles des élèves. Canadian Journal of Education/Revue canadienne de l'éducation, 35(1), 82.

Galand, B. \& Vanlede, M. (2004). Le sentiment d'efficacité personnelle dans l'apprentissage et la formation. Quel rôle jouet-il ? D'où vient-il? Comment intervenir? Savoirs, 5 (hors série), 91-116.

Jelassi, K., \& Herault, S. (2015). Continuité d'usage et appropriation de l'Internet mobile : un essai de modélisation. Management Avenir, 4, 59-77.

Klassen, R. M., Bong, M., Usher, E. L., Chong, W. H., Huan, V. S., Wong, I. Y., \& Georgiou, T. (2009). Exploring the validity of a teachers' self-efficacy scale in five countries. Contemporary educational psychology, 34(1), 67-76.

Klassen, R. M., \& Chiu, M. M. (2010). Effects on teachers' self- efficacy and job satisfaction: Teacher gender, years of experience, and job stress. Journal of Educational Psychology, 102 (3), 741-756. 
Olsommer, Z. \& Voyame-Egger, L. (2015). Le sentiment d'auto-efficacité des enseignants spécialisés en dernière année de formation. Thèse de doctorat. Haute école pédagogique du canton de Vaud.

Pintrich, P. R., et Schunk, D. H. (2002). Motivation in education: theory, research, and applications (2nd ed.). Upper Saddle River, NJ : Prentice Hall.

Rey, J., \& Coen, P. F. (2012). Évolutions des attitudes motivationnelles des enseignants pour l'intégration des technologies de l'information et de la communication. Formation et profession: Revue scientifique internationale en éducation, 20(2), 26-44.

Seegers, G., et Boekaerts, M. (1993). Task motivation and mathematics achievement in actual task situations. Learning and Instruction, 3(2), 133-150.

Trestini, M. (2012). Causes de non-usage des TICE à l'Université : des changements? Cas des enseignants du supérieur. Recherches Ẽ éducations, 6, 15-33. https://doi.org/10.4000/rechercheseducations. 935

Tricot, A., Plégat-Soutjis, F., Camps, J.-F., Amiel, A., Lutz, G. \& Morcillo, A., (2003). Utilité, utilisabilité, acceptabilité : interpréter les relations entre trois dimensions de l'évaluation des EIAH, Dans C. Desmoulins, P. Marquet, \& D. Bouhineau (Eds.), Actes de la conférence européenne sur les EIAH (p. 391-402), Strasbourg, 15-17 avril 2003. Paris, France : ATIEF, INRP.

Tschannen-Moran, M., Hoy, A. W., \& Hoy, W. K. (1998). Teacher efficacy: Its meaning and measure. Review of Educational Research, 68(2), 202-248. https://doi.org/10.3102/00346543068002202

Tschannen-Moran, M., \& Hoy, A. W. (2001). Teacher efficacy: Capturing an elusive construct. Teaching and teacher education, 17(7), 783-805.

Valls, M., \& Bonvin, P. (2015). Auto-efficacité des enseignants : quels outils d'évaluation utiliser? Mesure et évaluation en éducation, 38(3), 1-47.

Venkatesh, V., \& Bala, H. (2008). Technology acceptance model 3 and a research agenda on interventions. Decision sciences, 39(2), 273-315.

Venkatesh V. Et Davis F.-D. (2000). A theoretical extension of the technology acceptance model : four longitudinal field studies, Management Science, 46(2), 186-204.

Viel-Ruma, K., Houchins, D., Jolivette, K., \& Benson, G. (2010). Efficacy beliefs of special educators: The relationships among collective efficacy, teacher self-efficacy, and job satisfaction. Teacher Education and Special Education: The Journal of the Teacher Education Division of the Council for Exceptional Children, 33(3), 225-233.

Wolters, C. A., Daugherty, S. G. (2007). Goal structures and teacher's sense of efficacy: Their relation and association to teaching experience and academic level. Journal of Educational Psychology, 99(1), 181-193. https://doi.org/10.1037/00220663.99.1.181 